\title{
The effect of acute and 7-days dietary nitrate on mechanical efficiency, exercise performance and cardiac biomarkers in patients with chronic obstructive pulmonary disease
}

Citation for published version (APA):

Beijers, R. J. H. C. G., Huysmans, S. M. D., van de Bool, C., Kingma, B. R. M., Verdijk, L. B., van Loon, L. J. C., Meex, S. J. R., Gosker, H. R., \& Schols, A. M. W. J. (2018). The effect of acute and 7-days dietary nitrate on mechanical efficiency, exercise performance and cardiac biomarkers in patients with chronic obstructive pulmonary disease. Clinical Nutrition, 37(6), 1852-1861.

https://doi.org/10.1016/j.clnu.2017.10.011

Document status and date:

Published: 01/12/2018

DOI:

10.1016/j.clnu.2017.10.011

Document Version:

Publisher's PDF, also known as Version of record

Document license:

Taverne

Please check the document version of this publication:

- A submitted manuscript is the version of the article upon submission and before peer-review. There can be important differences between the submitted version and the official published version of record. People interested in the research are advised to contact the author for the final version of the publication, or visit the DOI to the publisher's website.

- The final author version and the galley proof are versions of the publication after peer review.

- The final published version features the final layout of the paper including the volume, issue and page numbers.

Link to publication

\footnotetext{
General rights rights.

- You may freely distribute the URL identifying the publication in the public portal. please follow below link for the End User Agreement:

www.umlib.nl/taverne-license

Take down policy

If you believe that this document breaches copyright please contact us at:

repository@maastrichtuniversity.nl

providing details and we will investigate your claim.
}

Copyright and moral rights for the publications made accessible in the public portal are retained by the authors and/or other copyright owners and it is a condition of accessing publications that users recognise and abide by the legal requirements associated with these

- Users may download and print one copy of any publication from the public portal for the purpose of private study or research.

- You may not further distribute the material or use it for any profit-making activity or commercial gain

If the publication is distributed under the terms of Article $25 \mathrm{fa}$ of the Dutch Copyright Act, indicated by the "Taverne" license above, 
Randomized Control Trials

\title{
The effect of acute and 7-days dietary nitrate on mechanical efficiency, exercise performance and cardiac biomarkers in patients with chronic obstructive pulmonary disease
}

\author{
Rosanne J.H.C.G. Beijers ${ }^{a}{ }^{*}$, Stephanie M.D. Huysmans ${ }^{\text {a }}$, Coby van de Bool ${ }^{\text {a }}$, \\ Boris R.M. Kingma ${ }^{\text {b, c }}$, Lex B. Verdijk ${ }^{\text {b }}$, Luc J.C. van Loon ${ }^{\text {b }}$, Steven J.R. Meex ${ }^{d}$, \\ Harry R. Gosker ${ }^{a}$, Annemie M.W.J. Schols ${ }^{\text {a }}$ \\ a Department of Respiratory Medicine, NUTRIM School of Nutrition and Translational Research in Metabolism, Maastricht University Medical Centre+, \\ Maastricht, The Netherlands \\ ${ }^{\mathrm{b}}$ Department of Human Biology and Movement Sciences, NUTRIM School of Nutrition and Translational Research in Metabolism, Maastricht University \\ Medical Centre+, Maastricht, The Netherlands \\ ${ }^{\mathrm{c}}$ TNO, Netherlands Organization for Applied Scientific Research, Defense, Safety \& Security, Soesterberg, The Netherlands \\ ${ }^{\mathrm{d}}$ Department of Clinical Chemistry, Cardiovascular Research Institute Maastricht (CARIM), Maastricht University Medical Centre+, Maastricht, The \\ Netherlands
}

\section{A R T I C L E I N F O}

\section{Article history:}

Received 13 July 2017

Accepted 19 October 2017

\section{Keywords:}

Blood pressure

COPD

Cardiac biomarkers

Dietary nitrate and mechanical efficiency

\section{S U M M A R Y}

Background \& aims: Many COPD patients have a reduced exercise capacity and mechanical efficiency and are at increased cardiometabolic risk. This study aimed to assess acute and 7-days effects of dietary nitrate on mechanical efficiency, exercise performance and cardiac biomarkers in patients with COPD. Methods: This double-blind, randomized cross-over placebo controlled trial included 20 mild-tomoderate COPD patients $(66.6 \pm 7.5$ years $)$ with moderate exercise impairments and decreased mechanical efficiency, normal BMI $\left(26 \pm 3 \mathrm{~kg} / \mathrm{m}^{2}\right)$ but high prevalence of abdominal obesity (83.3\%). Subjects were randomly allocated to the treatment order of 7 days sodium nitrate ingestion $(\sim 8 \mathrm{mmol} /$ day $)$ and 7 days placebo ( $\mathrm{NaCl}$ solution) or vice versa, separated by a washout period. Before (Day-1) and after (Day-7) both intervention periods resting metabolic rate and the metabolic response during submaximal cycle ergometry, cycling endurance time, plasma nitrate and nitrite levels, cardiac plasma biomarkers (e.g. cardiac troponin T, Nt-proBNP and creatinine kinase) and blood pressure were measured. Subsequently, gross, net and delta mechanical efficiency were calculated.

Results: Plasma nitrate and nitrite concentrations increased at Day- 1 and Day- 7 after sodium nitrate but not after placebo ingestion. Systolic and diastolic blood pressure did not change following nitrate ingestion. Furthermore, no differences were observed in gross, net, and delta mechanical efficiency during submaximal exercise, cycling endurance time and cardiac biomarkers between nitrate and placebo on Day-1 and Day-7. Meta-analysis of all available studies in COPD also showed no beneficial effect of beetroot juice on systolic and diastolic blood pressure.

Conclusion: Acute as well as 7-days sodium nitrate supplementation does not modulate mechanical efficiency, blood pressure or cardiac biomarkers in mild-to-moderate COPD patients.

๑ 2017 Elsevier Ltd and European Society for Clinical Nutrition and Metabolism. All rights reserved.

Abbreviations: BMD, bone mineral density; BMI, body mass index; BRJ, beetroot juice; CK, creatinine kinase; CKD-EPI, estimated glomerular filtration rate; COPD, chronic

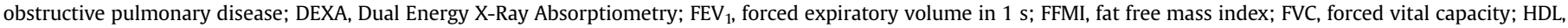

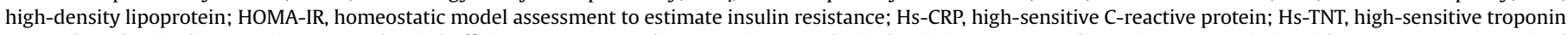

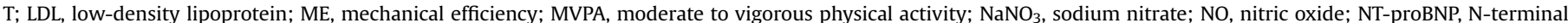

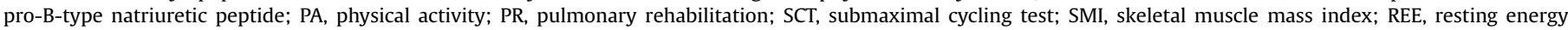
expenditure; RER, respiratory exchange rate; $\mathrm{VCO}_{2}$, carbon dioxide productions; $\mathrm{VO}_{2}$, oxygen consumption; Wmax, maximal work load.

* Corresponding author. P.O. Box 5800, 6202 AZ, Maastricht, The Netherlands. Fax: +0031 433875051.

E-mail address: r.beijers@maastrichtuniversity.nl (R.J.H.C.G. Beijers). 


\section{Introduction}

Chronic obstructive pulmonary disease (COPD) is a progressive lung disease characterized by persistent airflow obstruction resulting from enhanced inflammation in the airways [1]. Besides the respiratory impairment, extrapulmonary manifestations and comorbidities influence disease burden and mortality [1]. Common comorbidities include cardiovascular disease and metabolic syndrome [2,3]. Cardiometabolic risk is not only increased in obese patients but also in normal weight COPD patients with low muscle mass and abdominal obesity [4,5]. Furthermore, patients with COPD have lower mechanical efficiency, i.e. the proportion of work accomplished to energy expended, compared to healthy controls [6-8], possibly due to an increased oxygen cost of breathing [6] and impaired muscle mitochondrial metabolism [8-11]. As a lower mechanical efficiency can contribute to impaired exercise performance and hamper efficacy of aerobic exercise training, patients with COPD might benefit from interventions targeting mechanical efficiency.

Nitrate is an interesting nutrient that might improve both mechanical efficiency and cardiovascular health in COPD. Dietary nitrate is reduced to nitrite which subsequently is converted to nitric oxide (NO) [12]. As a result of dietary nitrate intake, NO availability will be increased which can have vasodilatory effects that can lower blood pressure and affect body temperature [13]. Indeed, two metaanalyses showed lowered blood pressure in adults with or without comorbidities after inorganic nitrate or beetroot juice (BRJ) supplementation [14,15]. Besides beneficial effects on blood pressure, increased NO availability can modulate muscle-related processes including muscle contractility, glucose homeostasis, blood flow, mitochondrial respiration and biogenesis [16]. A side effect of an increased blood flow in the extremities is that it may warm the skin and facilitate extra heat loss, which has to be compensated by a higher metabolic rate, or may lead to a reduction in body core temperature. Recently, a meta-analysis demonstrated improved endurance exercise performance following dietary nitrate ingestion in healthy adults [17]. Furthermore, oxygen cost of exercise has been shown to decrease after dietary nitrate intake, without affecting resting metabolic rate [18]. We therefore hypothesized that dietary nitrate might also modulate mechanical efficiency in COPD.

Dietary nitrate is mostly present in green leafy and root vegetables and in most research it is applied in the form of BRJ. Although beneficial physiological effects of BRJ are ascribed to the high nitrate content [19], it could be argued that nitrate is not the active nutrient after all or that nitrate interacts with other compounds in $\mathrm{BRJ}$ which cause the beneficial effects. In order to investigate the effects of nitrate alone, without needing to account for unknown interactions with other interventional compounds in the solution, sodium nitrate would be preferred. In healthy adults, a multiple dosing day strategy was more efficacious for improving exercise performance than an acute dose [20]. If this is also the case in patients with COPD it would be interesting to compare the acute effect with the multiple dosing day effects. We hypothesized that sodium nitrate modulates mechanical efficiency, improves exercise performance and improves cardiac biomarkers. Therefore, the aim of this study was to assess the acute and 7-days effects of sodium nitrate supplementation on mechanical efficiency, exercise performance and cardiac biomarkers in patients with COPD.

\section{Methods}

\subsection{Study design and subjects}

This study was a double-blind, randomized cross-over placebo controlled trial including 20 clinically stable COPD patients with a decreased mechanical efficiency based on screening of the ratio between peak oxygen consumption $\left(\mathrm{VO}_{2}\right)$ /maximal work load (Wmax) during incremental cycling test $(\geq 10 \mathrm{~mL} / \mathrm{min} / \mathrm{W})$ [21]. Patients were recruited via advertisements in local newspapers, between 2015 and 2016. Exclusion criteria were sodium intake limitation, long-term oxygen therapy, severe renal impairment (glomerular filtration rate $<30 \mathrm{~mL} / \mathrm{min}$ ), medications that might develop a risk for hypotension in combination with nitrate (i.e. PDE-5 inhibitors and nitrate-containing/releasing medication) and contra-indications for performing (sub-)maximal cycle test. The study was registered at clinicaltrials.gov (NCT02084758) and was approved by the Medical Ethics Committee from Maastricht University Medical Centre + (MUMC+ [NL47701.068.1/MEC 14-3016]). All patients gave their written informed consent.

\subsection{Supplementation protocol}

Before the start of the study, subjects visited the laboratory for a screening to measure lung function using forced spirometry (Masterlab, Jaeger, Würzburg, Germany) and to perform an incremental cycling ergometry test on an electromagnetic braked cycle ergometer (Ergoselect 200, Ergoline, Blitz, Germany) (see supplemental material for detailed methodology). Eligible subjects were randomly allocated to the treatment order of ingesting a daily dose of sodium nitrate $\left(\mathrm{NaNO}_{3}\right.$ [BASF, Ludwigshafen, Germany]) and placebo ( $\mathrm{NaCl}$ [Frisia Zout $\mathrm{BV}$, Harlingen, The Netherlands) dissolved in $140 \mathrm{~mL}$ water. Randomisation was performed by an independent researcher from the MUMC + and both subjects and researchers were blinded for the treatments till the end of the study. The nitrate intervention period consisted of a daily dose of $680 \mathrm{mg} \mathrm{NaNO}$ (which equals $496 \mathrm{mg}$ or $\sim 8 \mathrm{mmol}$ of nitrate) ingestion for 7 days. The placebo was provided in an equal daily dose of $680 \mathrm{mg} \mathrm{NaCl}$ ingestion for 7 days. Both intervention periods were separated by at least 7 days wash-out (Fig. 1). The first supplemental bolus was consumed in the laboratory at the first test-day, $2.5 \mathrm{~h}$ before the submaximal cycling test (Fig. 2). The last bolus was consumed in the laboratory at day 7 , also $2.5 \mathrm{~h}$ before the submaximal cycling test. Test-days were separated by the subjects consuming a supplemental bolus for 5 consecutive days.

Subjects were requested to abstain from foods naturally high in nitrate and to avoid using antibacterial mouthwash during the intervention periods [22].

\subsection{Test-days}

On the morning of each test-day, subjects came to the laboratory in a fasted state. Subjects orally ingested a temperature telemetry medical grade capsule (EQ02 SEW, Philips Respironic Massachusetts, USA) to measure the core temperature and afterwards iButton ${ }^{\circledR}$ dataloggers (DS1923, Maxim USA) were attached to 20 skin sites to measure the mean skin temperature. Subsequently, the ventilated hood system (Omnical; Maastricht University, Maastricht, The Netherlands) was used to measure resting energy expenditure (REE), an automated blood pressure monitor was used to measure the blood pressure (Omron Healthcare Inc, Field Court Lake Forest, USA) and an intravenous cannula was inserted into an antecubital vein to obtain a fasted blood sample (T0). After this, subjects received their supplemental beverage (Fig. 2) and one hour later they received a standardized liquid breakfast (125 mL Respifor, Nutricia, Zoetermeer, Netherlands). A submaximal cycle ergometry test at $50 \%$ Wmax for $10 \mathrm{~min}$, at a fixed pedal rate of 60-70 RPM was performed $2.5 \mathrm{~h}$ after ingestion of the beverage. After $10 \mathrm{~min}$, workload was increased to $70 \%$ Wmax and subjects were instructed to cycle until (symptom 


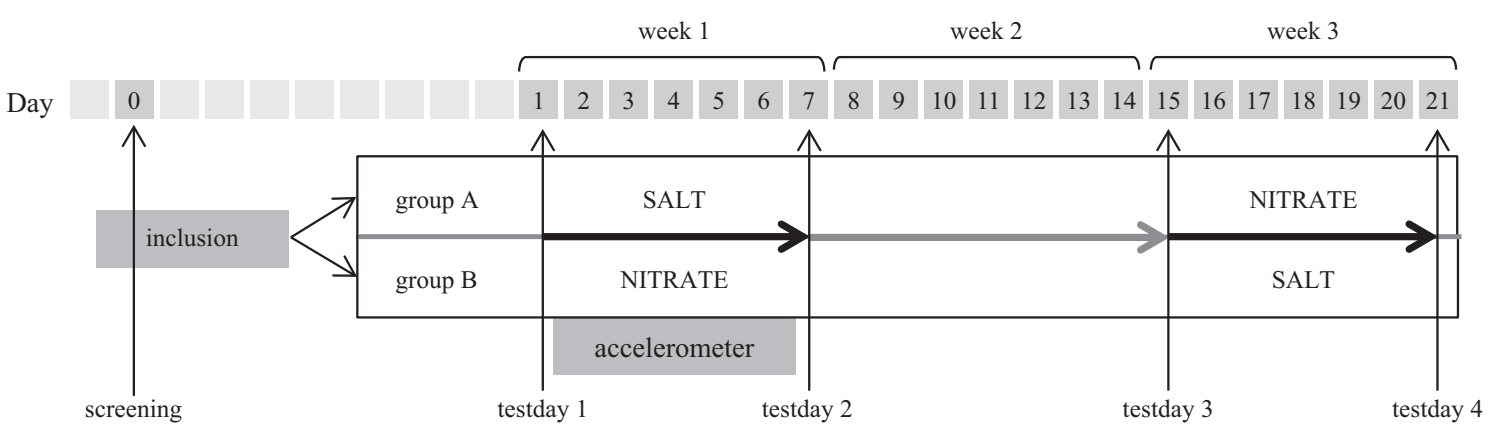

Fig. 1. Schematic illustration of the study protocol.

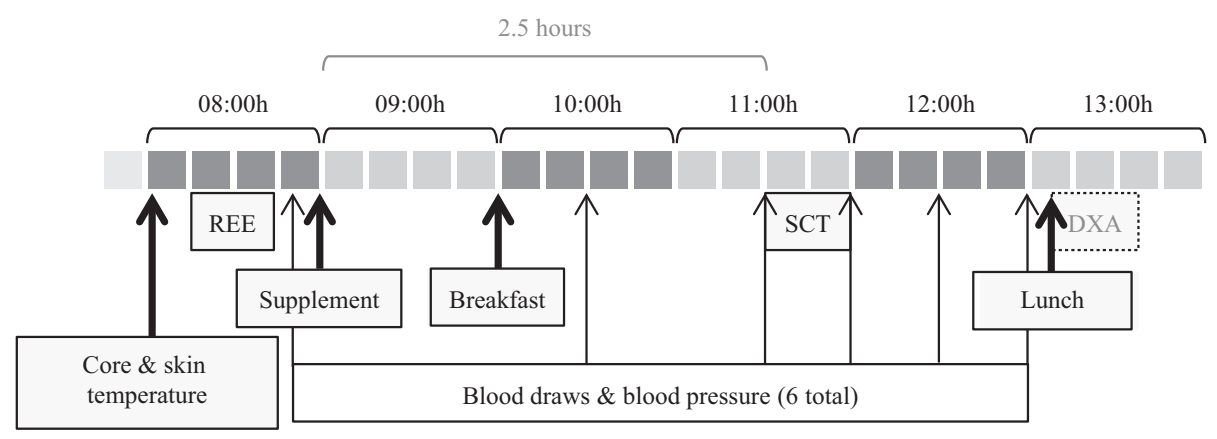

Fig. 2. Schematic illustration of the test-day. Abbreviations: REE, Resting energy expenditure; SCT, submaximal cycling test; DXA, Dual-energy X-ray absorptiometry.

limited) exhaustion with a maximum of 20 min. Gross, net, and delta mechanical efficiency were calculated according to Ettema et al. [23]. The abbreviated Weir formula was used to calculate energy expenditure during exercise [24]. Repeated blood pressure measurements and blood draws were performed 90 (T1) and 150 (T2) min after the beverage ingestion, immediately after the cycle test (T3), and subsequently after 30 (T4) and $60 \mathrm{~min}$ (T5).

Only on the first test-day Dual Energy X-Ray Absorptiometry (DEXA, Hologic, Discovery A, QDR Series, Bedford, MA, USA) was applied to assess body composition. During the 5 supplementation days, patients wore an accelerometer to verify similar physical activity (PA) levels during both intervention periods.

\subsection{Plasma analysis procedures}

Blood was sampled in Lithium-Heparin S-Monovette ${ }^{\circledR}$ tubes (Sarstedt, Nümbrecht, Germany). Tubes were immediately centrifuged at $1000 \mathrm{~g}$ for $10 \mathrm{~min}$, at $4{ }^{\circ} \mathrm{C}$ after which the aliquots were snap-frozen in liquid nitrogen and stored at $-80^{\circ} \mathrm{C}$ for subsequent analysis of plasma nitrate and nitrite using gas-phase chemiluminescence technique as was previously described [25]. Briefly, nitrate and nitrite concentrations were determined based on their reduction to NO. Upon the NO reaction with ozone, nitrogen dioxide is formed and during this production NO is quantified by detecting the light emitted using a thermoelectrically cooled, redsensitive photomultiplier tube, housed in a gas-phase chemiluminescence NO analyzer (Sievers Instruments, NOA ${ }^{\mathrm{TM}} 280 \mathrm{i}$, Analytix). Furthermore, plasma was used to determine cardiac biomarkers high sensitive troponin $\mathrm{T}$ (Hs-TNT), N-terminal pro-Btype natriuretic peptide (NT-proBNP) and creatine kinase (CK) (see supplemental material for details). Furthermore, baseline glucose and lipid profiles, estimated glomerular filtration rate (CKD-EPI) and high-sensitive C-reactive protein (Hs-CRP) were determined.

\subsection{Statistics}

Mechanical efficiency and temperature data were analyzed by two-way repeated measures ANOVA with treatment (nitrate and placebo) and test-day (day 1 and day 7) as within subject factors. Statistical analysis of all plasma and blood pressure data were performed using three-way repeated measures ANOVA with treatment (nitrate and placebo), test-day (day 1 and day 7) and time (T0, T1, T2, T3, T4 and T5) as within subject factors. All data were analyzed using Statistical Package for the Social Sciences (SPSS version 22 for Windows, IBM Corp., Armonk, USA) and data are presented as mean $\pm \mathrm{SD}$. A $p$-value $<0.05$ was considered statistically significant.

In order to compare results of the current study with previous literature in COPD a meta-analysis was performed as described in the supplemental material. Briefly, Pubmed database was used to find relevant articles on dietary nitrate in COPD. Data on systolic and diastolic blood pressure of both nitrate and placebo group were extracted. If a study did not show mean \pm SD for the suggested outcomes, original authors were contacted for additional information. Subsequently, standardized mean differences (95\% confidence intervals $(\mathrm{CI})$ ) have been calculated as Hedges' g due to the small sample sizes in the studies. A random effects model was used because of the considerable variability in several experimental factors (e.g. dose, duration and measurement) across studies [26]. The meta-analysis was performed with the Stata software package (StataCorp. 2015. Stata Statistical Software: Release 14. College Station, TX: StataCorp LP).

\section{Results}

In total 20 subjects were randomized in the study and 18 eventually finished the full study (Fig. 3). Subject characteristics are presented in Table 1 . The study population comprised a normal to 
overweight group (BMI $25.9 \pm 3.4 \mathrm{~kg} / \mathrm{m}^{2}$ ) with mild-to-moderate COPD (FEV ${ }_{1}$ pred $69.2 \pm 16.3$ ). Baseline gross mechanical efficiency during the incremental cycling test was $21.3 \pm 3.4 \%$, which was significantly lower than a healthy age matched control group from a previous study of our group ( $\mathrm{FEV}_{1}: 113.3 \pm 14.6 \%$ predicted, gross mechanical efficiency: $24.8 \pm 6.1 \%, p=0.049$ ) [10]. Abdominal obesity was present in $83.3 \%$ of the patients and four patients had low muscle mass. Plasma markers of lipid and glucose metabolism, kidney function and Hs-CRP were within normal range. Subjects reported that they had consumed all doses of the supplements. Both supplements were well-tolerated and no patients reported any deleterious side effects.

\subsection{Plasma nitrate and nitrite}

Baseline plasma concentration of nitrate $(58 \pm 36 \mu \mathrm{M}, \mathrm{p}=0.006)$ was $\sim 2$-fold increased at Nitrate day $7(133 \pm 106 \mu \mathrm{M})$, while no differences were observed in plasma nitrite (Fig. 4). Following ingestion of nitrate, plasma nitrate increased to the same extent at Nitrate day 1 (T0 vs T1; $331 \pm 54 \mu \mathrm{M}, p<0.001$ ) and day 7 (T0 vs T1; $339 \pm 57 \mu \mathrm{M}, p<0.001)$ and remained elevated throughout the test-day. Baseline plasma nitrite was $\sim 2$-fold increased after the nitrate ingestion at Nitrate day 1 (T0 to T1; $256 \pm 132$ to $634 \pm 345 \mathrm{nM} ; p<0.001$ ) as well as Nitrate day 7 (T0 vs T1: $245 \pm 165$ to $501 \pm 358 \mathrm{nM} ; p=0.003$ ) and also remained elevated throughout the test-day. Plasma nitrate and nitrite levels were not different at Placebo day 1 and 7.

\subsection{Submaximal cycling, resting energy expenditure and body temperatures}

During cycling at 50\% and 70\% Wmax, baseline gross mechanical efficiency (Placebo day 1 ) was $15.9 \pm 2.8 \%$ and $17.6 \pm 2.8 \%$ and baseline net mechanical efficiency was $20.4 \pm 3.0 \%$ and $21.4 \pm 2.9 \%$, respectively. Following nitrate ingestion, both gross and net mechanical efficiency were not different at Nitrate day 1 and day 7 , both at $50 \%$ and $70 \%$ Wmax, compared to placebo (Table 2). Delta mechanical efficiency was also not different between the nitrate and placebo treatment. Furthermore, no differences in $\mathrm{VO}_{2}, \mathrm{VCO}_{2}$, respiratory exchange rate and energy expenditure in rest as well as during cycling at $50 \%$ and $70 \%$ Wmax were observed between nitrate and placebo, both at day 1 and day 7 . Furthermore, the cycling endurance time was not different between the interventions. Skin and core temperature during the REE measurement and the submaximal cycling test were not different between nitrate and placebo at both day 1 and day 7 .

\subsection{Blood pressure}

Baseline systolic blood pressure was $140 \pm 14 \mathrm{mmHg}$ and baseline diastolic blood pressure was $81 \pm 10 \mathrm{mmHg}$. Following acute and 7-days nitrate ingestion blood pressure as well as heart rate did not change compared to placebo (Table 3). At both Nitrate and Placebo day 1 and day 7, systolic blood pressure decreased significantly (T0 vs T4; $-13 \pm 12 \mathrm{mmHg}, p<0.001$ ) during the

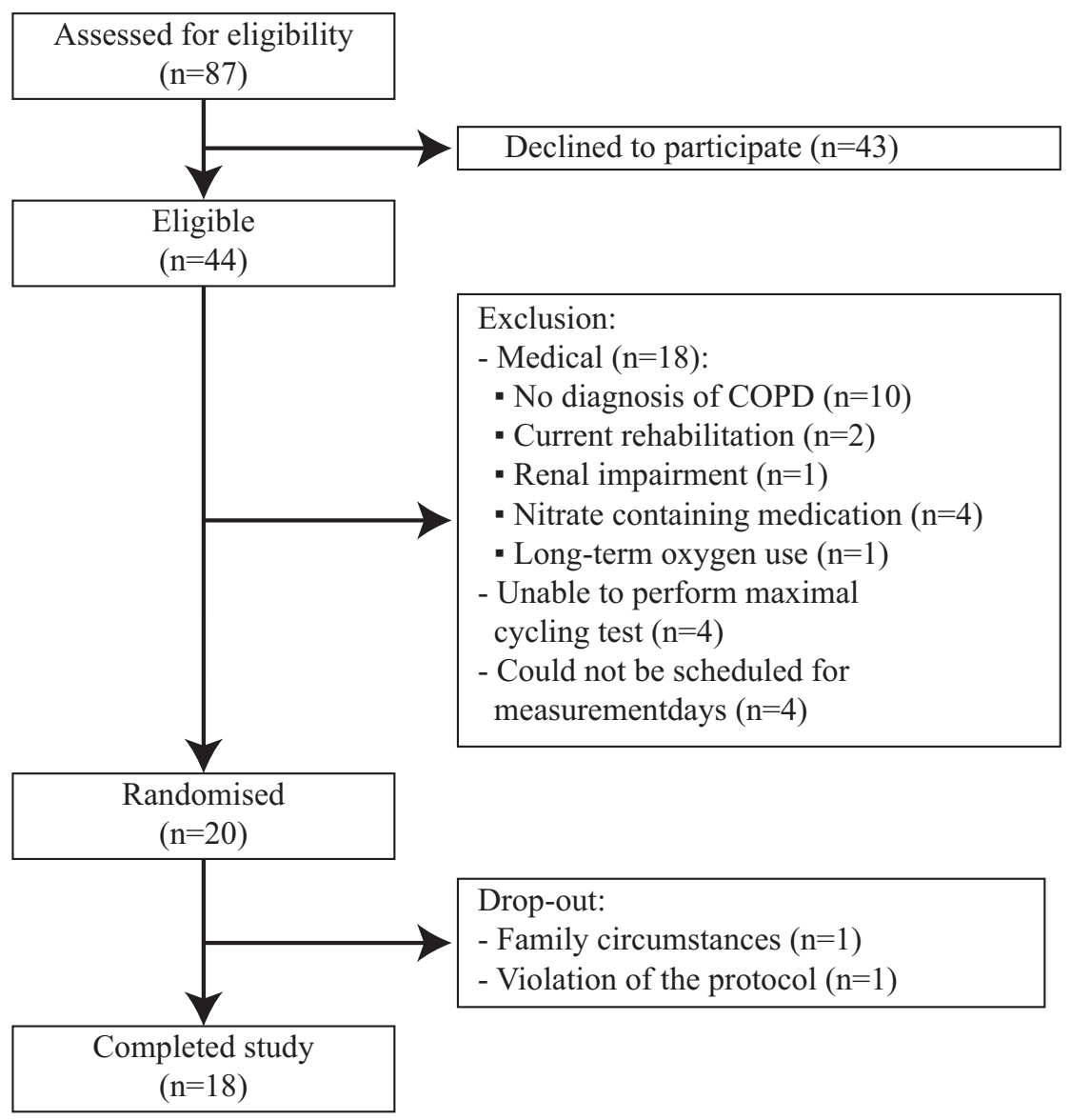

Fig. 3. Inclusion flowchart. 
Table 1

Subject characteristics $(\mathrm{n}=18)$.

\begin{tabular}{|c|c|}
\hline & $\mathrm{N}=18$ \\
\hline Age, y & $66.6 \pm 7.5$ \\
\hline Males, n (\%) & $13(72.2)$ \\
\hline \multicolumn{2}{|l|}{ Smoking status } \\
\hline Current smokers, n (\%) & $8(44.4)$ \\
\hline Former smokers, n (\%) & $10(55.6)$ \\
\hline $\mathrm{FEV}_{1}, \%$ pred & $69.2 \pm 16.3$ \\
\hline FVC, \%pred & $97.8 \pm 18.5$ \\
\hline $\mathrm{FEV}_{1} / \mathrm{FVC}, \%$ & $54.2 \pm 9.8$ \\
\hline BMI, $\mathrm{kg} / \mathrm{m}^{2}$ & $25.9 \pm 3.4$ \\
\hline FFMI, $\mathrm{kg} / \mathrm{m}^{2}$ & $17.3 \pm 1.9$ \\
\hline SMI, $\mathrm{kg} / \mathrm{m}^{2}$ & $7.3 \pm 0.9$ \\
\hline Low SMI, n (\%) & $4(22.2)$ \\
\hline Fat percentage, $\%$ & $30.7 \pm 5.6$ \\
\hline Abdominal obesity, n (\%) & $15(83.3)$ \\
\hline $\mathrm{BMD}, \mathrm{g} / \mathrm{cm}^{2}$ & $1.1 \pm 0.1$ \\
\hline Peak $\mathrm{VO}_{2}, \mathrm{ml} / \mathrm{min} / \mathrm{kg}$ & $19.7 \pm 4.0$ \\
\hline Peak $\mathrm{VO}_{2}, \%$ pred & $79(70-87)$ \\
\hline Wmax, W & $115 \pm 34$ \\
\hline Wmax, \%pred & $76.1 \pm 18.9$ \\
\hline Maximal heart rate, beats/min & $136 \pm 17$ \\
\hline Cholesterol, $\mathrm{mmol} / \mathrm{L}$ & $4.87 \pm 0.92$ \\
\hline HDL cholesterol, mmol/L & $1.2(1.1-1.5)$ \\
\hline LDL cholesterol, mmol/L & $2.75 \pm 0.88$ \\
\hline Triglycerides, mmol/L & $1.2(0.9-1.7)$ \\
\hline Glucose, $\mathrm{mmol} / \mathrm{L}$ & $5.96(5.55-6.72)$ \\
\hline Insulin, $\mathrm{mU} / \mathrm{L}$ & $5.7 \pm 3.2$ \\
\hline HOMA-IR & $1.7 \pm 1.0$ \\
\hline Kreatinine, $\mu \mathrm{mol} / \mathrm{L}$ & $81.8 \pm 19.9$ \\
\hline CKD-epi, $\mathrm{ml} / \mathrm{min} / 1.73 \mathrm{~m}^{2}$ & $80.0 \pm 14.2$ \\
\hline Hs-CRP, mg/L & $2.6(0.9-6.0)$ \\
\hline
\end{tabular}

Data are shown as mean \pm SD unless indicated otherwise.

Abbreviations: BMD, bone mineral density; CKD-epi, Chronic Kidney Disease Epidemiology Collaboration; $\mathrm{FEV}_{1}$, forced expiratory volume in $1 \mathrm{~s}$; FFMI, fat free mass index; FVC, forced vital capacity; Hs-CRP, high-sensitive C-reactive protein; SMI, skeletal muscle mass index; $\mathrm{VO}_{2}$, oxygen consumption; Wmax, maximal work load.

resting period after the submaximal cycling test compared to baseline while diastolic blood pressure remained unchanged. Furthermore, heart rate was significantly increased (T0 vs T3; $41 \pm 15$ beats/min, $p<0.001$ ) directly after the submaximal cycling test and remained elevated during the resting period after cycling (T0 vs T4; $13 \pm 9$ and T0 vs T5; $8 \pm 7$ beats/min, respectively, both $p<0.001$ ) at both day 1 and 7 of nitrate and placebo ingestion.

\subsection{Cardiac biomarkers}

No differences were observed in cardiac markers Hs-TNT, NTproBNP and $\mathrm{CK}$ following acute and 7-days nitrate ingestion compared to placebo (Fig. 5). All cardiac markers were increased after exercise (T2 vs T3; Hs-TNT $0.4 \pm 0.1 \mathrm{ng} / \mathrm{mL}, p=0.011$; NTproBNP $1.7 \pm 0.5 \rho \mathrm{mol} / \mathrm{L}, p=0.003$; CK $8.1 \pm 1.7, p<0.001)$. Furthermore, Hs-TNT was higher at baseline (T0) compared to other time points at the test-day.

\subsection{Physical activity levels}

Total physical activity level as well as physical activity pattern was not different during nitrate and placebo ingestion (Table 4).

\subsection{Meta-analysis}

Main characteristics of the studies are summarized in Supplemental Table 1 . In total 94 patients participated in the included studies. The mean age range was 65-70 years and patients were normal to overweight (mean BMI: $25-29 \mathrm{~kg} / \mathrm{m}^{2}$ ) with mild-to-moderate COPD (mean $\mathrm{FEV}_{1}$ : 43-62 \%predicted). All
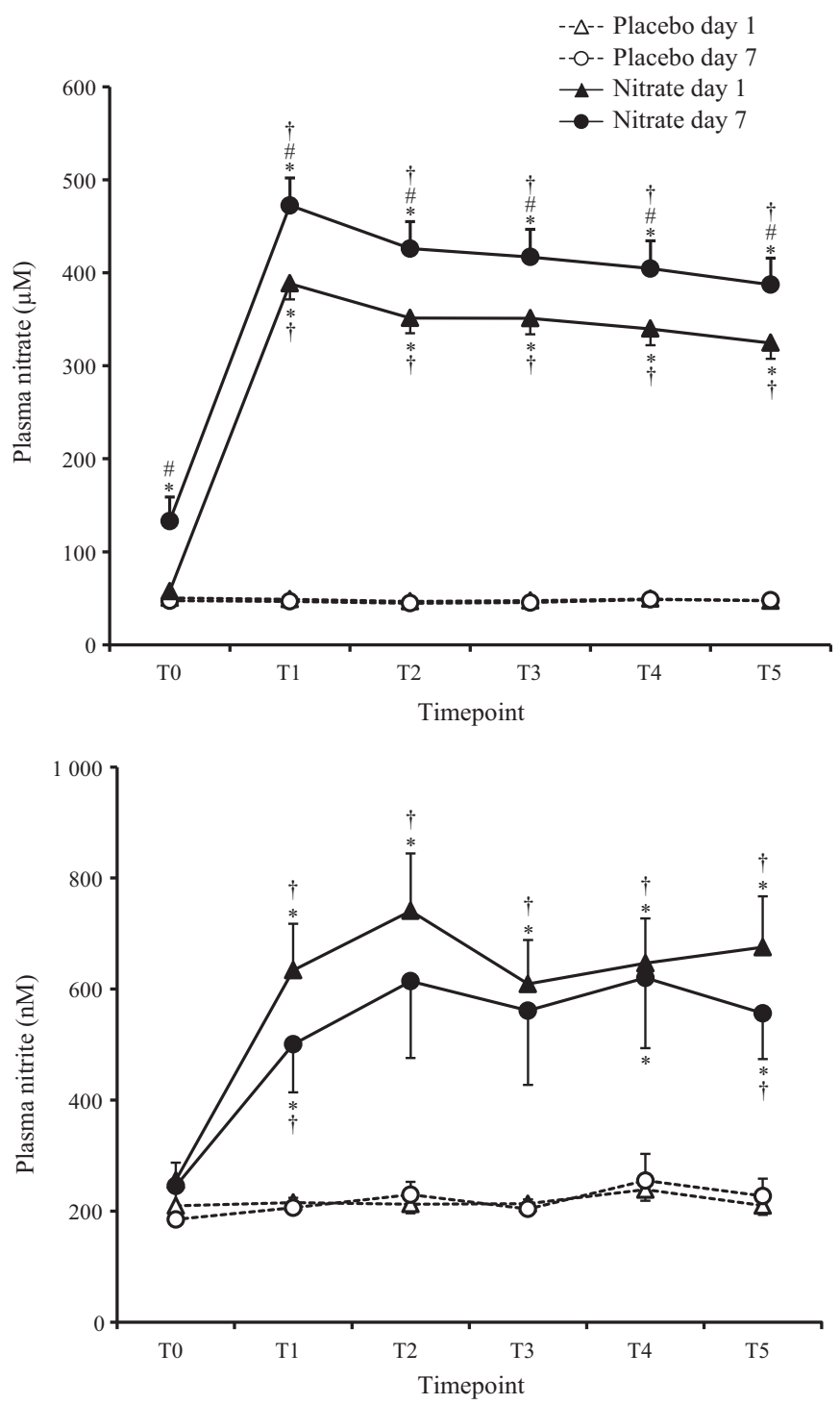

Fig. 4. Plasma nitrate and nitrite concentrations on day 1 and day 7 for the placebo and nitrate intervention at different timepoints. Blood draws were performed at baseline (T0), 90 (T1) and 150 (T2) minutes after the beverage ingestion, immediately after the cycle test (T3), and subsequently after 30 (T4) and $60 \mathrm{~min}$ (T5). Values are mean \pm SEM; $n=17$. *Significantly different from placebo $(\mathrm{p}<0.05)$. \#Significantly different from nitrate day 7 ( $p<0.05)$. †Significantly different from T0 $(p<0.05)$.

studies used beetroot juice as a nitrate-rich supplement, with a nitrate-dose ranging between 6.77 and $12.0 \mathrm{mmol} /$ day. Following the meta-analysis the standardized mean difference was -0.03 ( $95 \%$ CI -0.32 to 0.26 ) for systolic blood pressure and -0.24 (95\% CI -0.55 to 0.08 ) for diastolic blood pressure, showing a small but nonsignificant effect in favour of dietary nitrate (Fig. 6).

\section{Discussion}

To our knowledge this is the first study investigating the acute as well as the 7-days effects of sodium nitrate supplementation on mechanical efficiency and cardiac biomarkers in mild-to-moderate COPD patients. Acute as well as 7-days nitrate supplementation did not alter mechanical efficiency, despite clear and anticipated elevated plasma nitrate and nitrite levels. Furthermore, exercise performance, blood pressure, cardiac biomarkers Hs-TNT, NTproBNP and CK, skin and core temperatures and finally REE were 
Table 2

Steady-state values of energy expenditure during rest and submaximal cycling after nitrate and placebo ingestion.

\begin{tabular}{|c|c|c|c|c|c|}
\hline & \multicolumn{2}{|l|}{ Nitrate } & \multicolumn{2}{|l|}{ Placebo } & \multirow[t]{2}{*}{ p-value } \\
\hline & Day 1 & Day 7 & Day 1 & Day 7 & \\
\hline \multicolumn{6}{|l|}{ Resting energy expenditure } \\
\hline $\mathrm{VO}_{2}, \mathrm{~mL} / \mathrm{min}$ & $231 \pm 31$ & $233 \pm 31$ & $234 \pm 30$ & $237 \pm 34$ & 0.825 \\
\hline $\mathrm{VCO}_{2}, \mathrm{~mL} / \mathrm{min}$ & $196 \pm 28$ & $194 \pm 23$ & $193 \pm 21$ & $198 \pm 26$ & 0.062 \\
\hline $\mathrm{VO}_{2}, \mathrm{~mL} / \mathrm{min} / \mathrm{kg}$ & $3.0 \pm 0.3$ & $3.0 \pm 0.2$ & $3.0 \pm 0.2$ & $3.1 \pm 0.2$ & 0.823 \\
\hline $\mathrm{VCO}_{2}, \mathrm{~mL} / \mathrm{min} / \mathrm{kg}$ & $2.5 \pm 0.3$ & $2.5 \pm 0.3$ & $2.5 \pm 0.3$ & $2.6 \pm 0.3$ & 0.083 \\
\hline RER & $0.85 \pm 0.05$ & $0.84 \pm 0.05$ & $0.83 \pm 0.04$ & $0.84 \pm 0.05$ & 0.195 \\
\hline Energy expenditure, kcal/min & $1.12 \pm 0.15$ & $1.12 \pm 0.14$ & $1.13 \pm 0.14$ & $1.14 \pm 0.16$ & 0.538 \\
\hline Core temperature, ${ }^{\circ} \mathrm{C}^{\dagger}$ & $36.8 \pm 0.2$ & $36.8 \pm 0.3$ & $36.9 \pm 0.3$ & $36.7 \pm 0.6$ & 0.313 \\
\hline Skin temperature, ${ }^{\circ} \mathrm{C}$ & $32.9 \pm 0.5$ & $33.0 \pm 0.5$ & $33.0 \pm 0.4$ & $33.0 \pm 0.5$ & 0.827 \\
\hline \multicolumn{6}{|l|}{ Submaximal cycling at $\mathbf{5 0 \%} \mathbf{W m a x}$} \\
\hline $\mathrm{VO}_{2}, \mathrm{~mL} / \mathrm{min}$ & $1034 \pm 182$ & $1032 \pm 191$ & $1039 \pm 182$ & $1033 \pm 191$ & 0.806 \\
\hline $\mathrm{VCO}_{2}, \mathrm{~mL} / \mathrm{min}$ & $945 \pm 184$ & $950 \pm 195$ & $949 \pm 175$ & $953 \pm 180$ & 0.951 \\
\hline $\mathrm{VO}_{2}, \mathrm{~mL} / \mathrm{min} / \mathrm{kg}$ & $13.3 \pm 2.4$ & $13.3 \pm 2.5$ & $13.4 \pm 2.3$ & $13.3 \pm 2.2$ & 0.622 \\
\hline $\mathrm{VCO}_{2}, \mathrm{~mL} / \mathrm{min} / \mathrm{kg}$ & $12.2 \pm 2.4$ & $12.2 \pm 2.6$ & $12.2 \pm 2.3$ & $12.2 \pm 2.1$ & 0.761 \\
\hline RER & $0.91 \pm 0.04$ & $0.92 \pm 0.06$ & $0.91 \pm 0.04$ & $0.92 \pm 0.04$ & 0.744 \\
\hline Energy expenditure, kcal/min & $5.07 \pm 0.91$ & $5.07 \pm 0.96$ & $5.10 \pm 0.90$ & $5.08 \pm 0.94$ & 0.840 \\
\hline Gross ME, $\%$ & $16.0 \pm 2.8$ & $16.0 \pm 2.7$ & $15.9 \pm 2.8$ & $16.0 \pm 3.1$ & 0.626 \\
\hline Net ME, \% & $20.6 \pm 3.2$ & $20.6 \pm 2.8$ & $20.4 \pm 3.0$ & $20.7 \pm 3.4$ & 0.647 \\
\hline Core temperature, ${ }^{\circ} \mathrm{C}$ & $37.0 \pm 0.3$ & $36.9 \pm 0.4$ & $37.1 \pm 0.4$ & $36.7 \pm 0.7$ & 0.089 \\
\hline Skin temperature, ${ }^{\circ} \mathrm{C}$ & $31.8 \pm 0.4$ & $31.9 \pm 0.6$ & $31.9 \pm 0.6$ & $32.0 \pm 0.6$ & 0.651 \\
\hline \multicolumn{6}{|l|}{ Submaximal cycling at $70 \% \mathrm{Wmax}$} \\
\hline $\mathrm{VO}_{2}, \mathrm{~mL} / \mathrm{min}$ & $1302 \pm 274$ & $1315 \pm 291$ & $1319 \pm 273$ & $1323 \pm 275$ & 0.633 \\
\hline $\mathrm{VCO}_{2}, \mathrm{~mL} / \mathrm{min}$ & $1246 \pm 298$ & $1260 \pm 307$ & $1239 \pm 278$ & $1269 \pm 276$ & 0.506 \\
\hline $\mathrm{VO}_{2}, \mathrm{~mL} / \mathrm{min} / \mathrm{kg}$ & $16.7 \pm 3.7$ & $16.9 \pm 3.9$ & $16.9 \pm 3.6$ & $16.9 \pm 3.9$ & 0.558 \\
\hline $\mathrm{VCO}_{2}, \mathrm{~mL} / \mathrm{min} / \mathrm{kg}$ & $16.0 \pm 3.9$ & $16.1 \pm 4.0$ & $15.9 \pm 3.7$ & $16.2 \pm 3.5$ & 0.583 \\
\hline RER & $0.95 \pm 0.05$ & $0.95 \pm 0.05$ & $0.93 \pm 0.06$ & $0.96 \pm 0.04$ & 0.195 \\
\hline Energy expenditure, kcal/min & $6.45 \pm 1.39$ & $6.51 \pm 1.47$ & $6.51 \pm 1.37$ & $6.56 \pm 1.37$ & 0.868 \\
\hline Gross ME, $\%$ & $17.8 \pm 2.7$ & $17.6 \pm 2.6$ & $17.6 \pm 2.8$ & $17.5 \pm 3.0$ & 0.839 \\
\hline Net ME, \% & $21.7 \pm 2.8$ & $21.5 \pm 2.5$ & $21.4 \pm 2.9$ & $21.3 \pm 3.1$ & 0.757 \\
\hline Delta ME, \% & $26.9 \pm 4.8$ & $24.7 \pm 3.6$ & $26.6 \pm 6.8$ & $24.3 \pm 3.9$ & 0.997 \\
\hline Cycling time, min & $1186 \pm 402$ & $1316 \pm 440$ & $1331 \pm 426$ & $1300 \pm 387$ & 0.077 \\
\hline Core temperature, ${ }^{\circ} \mathrm{C}$ & $37.2 \pm 0.3$ & $37.2 \pm 0.4$ & $37.4 \pm 0.4$ & $37.0 \pm 0.9$ & 0.066 \\
\hline Skin temperature, ${ }^{\circ} \mathrm{C}$ & $32.0 \pm 0.5$ & $32.0 \pm 0.6$ & $32.0 \pm 0.4$ & $32.1 \pm 0.6$ & 0.763 \\
\hline
\end{tabular}

Data are shown as mean \pm SD.

Abbreviations: $\mathrm{ME}$, mechanical efficiency; $\mathrm{VO}_{2}$, oxygen consumption; $\mathrm{VCO}_{2}$, carbon dioxide production; RER, respiratory exchange rate.

Table 3

Blood pressure and heart rate after nitrate or placebo ingestion $(\mathrm{n}=18)$.

\begin{tabular}{|c|c|c|c|c|c|}
\hline & \multicolumn{2}{|l|}{ Nitrate } & \multicolumn{2}{|l|}{ Placebo } & \multirow[t]{2}{*}{ p-value } \\
\hline & Day 1 & Day 7 & Day 1 & Day 7 & \\
\hline \multicolumn{6}{|c|}{ Systolic blood pressure, mmHg } \\
\hline T0 & $137 \pm 15$ & $135 \pm 18$ & $137 \pm 15$ & $137 \pm 21$ & 0.613 \\
\hline $\mathrm{T} 1$ & $132 \pm 17$ & $130 \pm 18$ & $134 \pm 15$ & $138 \pm 21$ & 0.138 \\
\hline $\mathrm{T} 2$ & $135 \pm 19$ & $135 \pm 18$ & $135 \pm 18$ & $138 \pm 20$ & 0.655 \\
\hline T3 & $152 \pm 33$ & $148 \pm 22$ & $145 \pm 31$ & $151 \pm 26$ & 0.165 \\
\hline $\mathrm{T} 4$ & $123 \pm 19 *$ & $122 \pm 16^{*}$ & $124 \pm 17^{*}$ & $123 \pm 16^{*}$ & 0.820 \\
\hline T5 & $125 \pm 17^{*}$ & $125 \pm 15^{*}$ & $126 \pm 13^{*}$ & $130 \pm 15$ & 0.278 \\
\hline \multicolumn{6}{|c|}{ Diastolic blood pressure, $\mathrm{mmHg}$} \\
\hline T0 & $79 \pm 9$ & $78 \pm 11$ & $79 \pm 13$ & $79 \pm 11$ & 0.639 \\
\hline $\mathrm{T} 1$ & $74 \pm 10^{*}$ & $74 \pm 11$ & $75 \pm 10$ & $77 \pm 12$ & 0.368 \\
\hline $\mathrm{T} 2$ & $78 \pm 8$ & $78 \pm 9$ & $80 \pm 10$ & $78 \pm 10$ & 0.348 \\
\hline T3 & $81 \pm 13$ & $80 \pm 14$ & $77 \pm 11$ & $78 \pm 11$ & 0.635 \\
\hline $\mathrm{T} 4$ & $77 \pm 13$ & $73 \pm 9$ & $78 \pm 11$ & $76 \pm 10$ & 0.535 \\
\hline T5 & $76 \pm 11$ & $76 \pm 12$ & $77 \pm 10$ & $81 \pm 10$ & 0.188 \\
\hline \multicolumn{6}{|c|}{ Heart rate, beats/min } \\
\hline T0 & $64 \pm 8$ & $64 \pm 8$ & $64 \pm 8$ & $64 \pm 7$ & 0.862 \\
\hline $\mathrm{T} 1$ & $69 \pm 11$ & $68 \pm 11$ & $70 \pm *{ }^{*} *$ & $67 \pm 9$ & 0.236 \\
\hline $\mathrm{T} 2$ & $68 \pm 9^{*}$ & $68 \pm 10$ & $67 \pm 9$ & $67 \pm 10$ & 0.762 \\
\hline T3 & $106 \pm 12^{*}$ & $106 \pm 17^{*}$ & $106 \pm 13^{*}$ & $104 \pm 18^{*}$ & 0.676 \\
\hline $\mathrm{T} 4$ & $77 \pm 10^{*}$ & $76 \pm 10^{*}$ & $78 \pm 13^{*}$ & $77 \pm 12^{*}$ & 0.980 \\
\hline T5 & $72 \pm 10^{*}$ & $71 \pm 9^{*}$ & $72 \pm 13^{*}$ & $72 \pm 12^{*}$ & 0.771 \\
\hline
\end{tabular}

Measurements were performed at baseline (T0), 90 (T1) and 150 (T2) minutes after the beverage ingestion, immediately after the cycle test (T3), and subsequently after 30 (T4) and $60 \mathrm{~min}$ (T5).

*Significantly different from T0 $(\mathrm{p}<0.05)$. Values are mean $\pm S D ; n=18$. not affected by sodium nitrate. These findings were in contrast to our hypothesis.

We expected improved mechanical efficiency after sodium nitrate ingestion as nitrate is suggested to decrease $\mathrm{VO}_{2}$ during exercise in healthy subjects, without alterations in REE [18]. However, in the current study both mechanical efficiency and $\mathrm{VO}_{2}$ during submaximal exercise were not affected after sodium nitrate ingestion. Since most studies showing beneficial effects of dietary nitrate on oxygen requirements during exercise are performed in healthy young individuals it is possible that $\mathrm{VO}_{2}$ kinetics cannot be altered at an older age or in a clinically compromised older population. Two studies have been performed in healthy older subjects with conflicting results $[27,28]$. After nitrate rich BRJ, one study showed a reduced $\mathrm{VO}_{2}$ mean response time in the transition from standing rest to treadmill walking which might indicate a reduced reliance on nonoxidative metabolic processes across the transition from a lower to a higher metabolic rate [27]. However, the oxygen cost of exercise remained unchanged, corresponding to another study in which no changes in resting, submaximal and maximal $\mathrm{VO}_{2}$ during incremental cycling were found [28]. Furthermore, in older patients with heart failure [29], type 2 diabetes mellitus [30] and COPD [31-34] most studies could not find improved $\mathrm{VO}_{2}$ kinetics after BRJ ingestion. Only one study showed slightly decreased isotime $\mathrm{VO}_{2}$ during submaximal cycling following acute BRJ ingestion [32]. Since patients with COPD spend probably more time walking instead of cycling it could be questioned whether the cycling test was the most optimal test to find beneficial effects of 

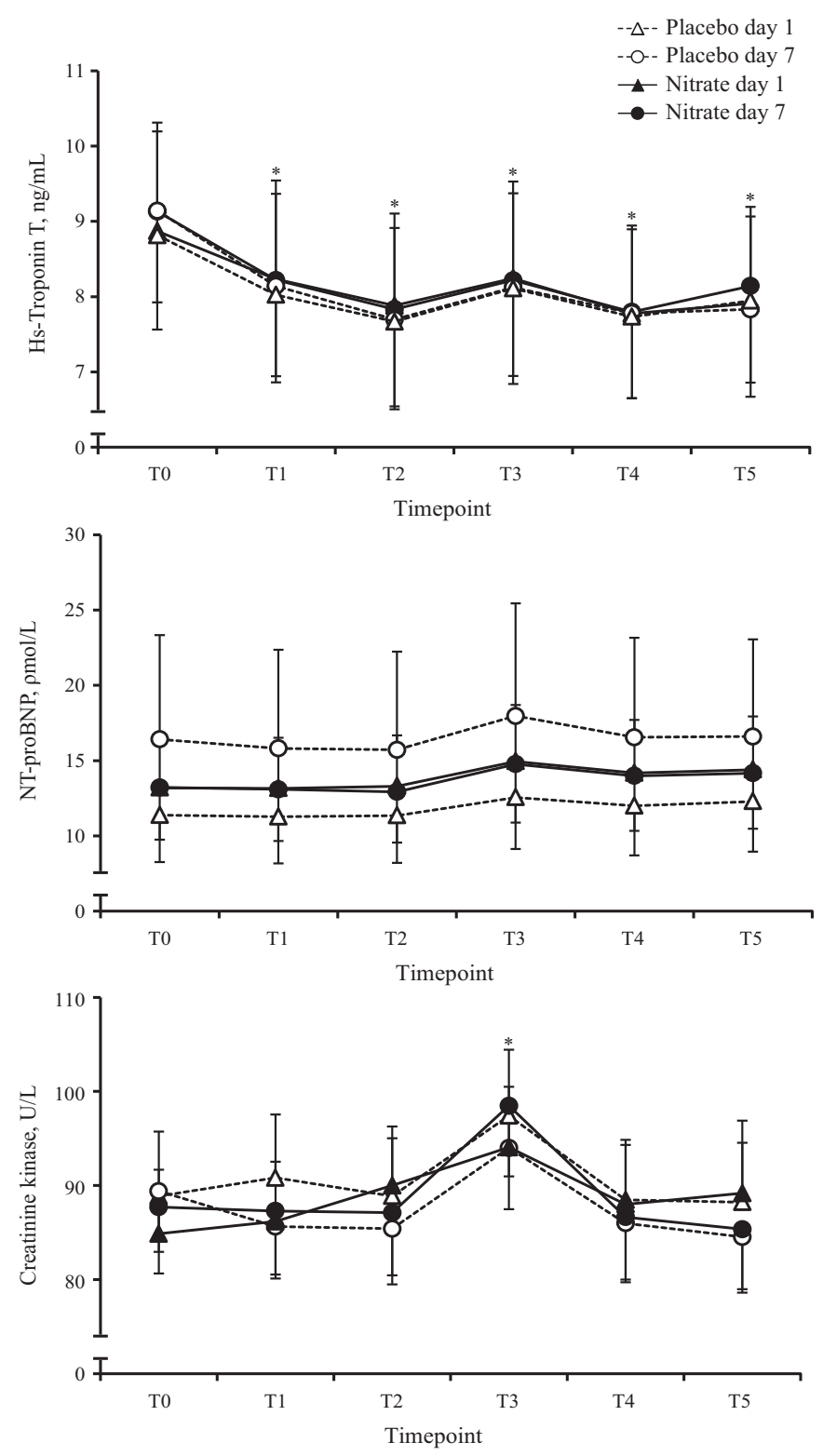

Fig. 5. Plasma high sensitive troponin T, NT-proBNP and creatinine kinase on day 1 and day 7 for the placebo and nitrate intervention at different timepoints. Blood draws were performed at baseline (T0), 90 (T1) and 150 (T2) minutes after the beverage ingestion, immediately after the cycle test (T3), and subsequently after 30 (T4) and $60 \mathrm{~min}(\mathrm{~T} 5)$. Values are mean $\pm \mathrm{SEM} ; \mathrm{n}=17$. No significant differences were observed between nitrate versus placebo and day 1 versus day 7 . *Significantly different from T0 ( $\mathrm{p}<0.05$ ). Abbreviation: NT-proBNP, N-terminal pro-B-type natriuretic peptide.

Table 4

Physical activity levels during nitrate and placebo intervention $(n=17)$.

\begin{tabular}{llll}
\hline & Placebo & Nitrate & p-value \\
\hline Total activity, counts/min & $204 \pm 106$ & $214 \pm 104$ & 0.528 \\
Time spent in sedentary PA, \% of wear time & $68.5 \pm 12.6$ & $68.7 \pm 9.6$ & 0.866 \\
Time spent in lifestyle PA, \% of wear time & $23.8 \pm 8.1$ & $23.2 \pm 5.4$ & 0.573 \\
Time spent in MVPA, \% of wear time & $7.8 \pm 5.4$ & $8.1 \pm 5.0$ & 0.605 \\
\hline
\end{tabular}

Data are shown as mean $\pm \mathrm{SD}$.

Abbreviations: PA, physical activity; MVPA, moderate to vigorous physical activity.

nitrate on mechanical efficiency in COPD. However, a previous study showed good reproducibility of the submaximal cycling test to measure mechanical efficiency in patients with COPD [6]. Furthermore, previous studies in COPD investigating the effect of dietary nitrate on walking performance showed no beneficial effect of dietary nitrate [33-36]. Overall, previous studies in COPD show no convincing improvements on $\mathrm{VO}_{2}$ kinetics after nitrate ingestion supporting the results of the current study (Supplemental Table 1).

Nitrate-rich supplements have increased in popularity among elite and recreational athletes as they have been shown to improve exercise capacity [17]. For this reason we hypothesized that nitrate could be a useful intervention in COPD as adjunct to pulmonary rehabilitation (PR) to increase the effects of exercise training. However, the current study showed no improvement in cycling endurance time. This corresponds to previous studies in COPD (Supplemental Table 1), in which almost no effects of BRJ were found on exercise performance. Only two studies showed significant improvements in submaximal cycling time and walking distance after acute ingestion of BRJ [31,35]. However, these studies used prune juice and blackcurrant cordial as placebo, which is not a robust placebo for BRJ. The effect of nitrate on exercise performance has been shown to be enhanced supplemented in combination with exposure to ultraviolet A radiation (UVA) and might be influenced by seasonal differences [37]. Despite this study showed no effect of nitrate on exercise performance in absence of UVA radiation there seemed to be a trend towards reduced oxygen consumption during exercise after nitrate ingestion without exposure to UVA radiation. Therefore, we believe differences in UVA radiation do not affect the results of the current study.

In the current study we recruited patients via advertisements, which resulted in inclusion of normal-weight mild-to-moderate COPD patients with moderate exercise impairment. Although patients were not referred to PR, all patients had a sedentary lifestyle corresponding to the PA levels of patients referred to outpatient PR [4]. Besides, patients had moderate decreased mechanical efficiency, which was significantly lower compared to healthy controls from a previous study by our group [10]. It has recently been shown that gross efficiency is declined in COPD with increasing disease severity [38], which might suggest that more severe diseased COPD patients might still benefit from dietary nitrate. However, in the current study the response of sodium nitrate intake on mechanical efficiency was not correlated with baseline mechanical efficiency (data not shown), suggesting the results would be similar in case more severe patients would be included. Therefore, we believe dietary nitrate is not the promising adjunct to PR to elevate the training effects.

It has been established that in healthy and mostly young subjects dietary nitrate supplementation can lower blood pressure $[14,15]$. In the current study, nitrate supplementation caused no changes in blood pressure and the meta-analysis also showed no beneficial effects of BRJ on blood pressure in the studies in COPD so far (Fig. 6). Results may be influenced by current use of antihypertensive medication. Two studies did not find significant reductions in blood pressure in older hypertensive subjects that were on antihypertensive medication $[39,40]$, suggesting that an additional reduction in blood pressure might not occur in a group of patients whose blood pressure is already well-controlled. In the current study 7 patients were on antihypertensive medication. However, excluding these patients from analyses did not influence the results (data not shown). Another possible explanation for the lack of changes in blood pressure might be the vascular ageing process in which the capacity to convert nitrate to NO is possibly reduced and the sensitivity of vascular smooth muscle cells to the vasodilatory effects of NO might be diminished [41]. This might also be the case in COPD and it could be speculated that higher doses of nitrate may be required to detect beneficial effects on blood pressure. Directly after the cycling test an increase in blood pressure was observed, while after this phase (T4 and T5) the systolic blood pressure was significantly lower compared to baseline (T0) which was consistent between both experimental groups at both days. 


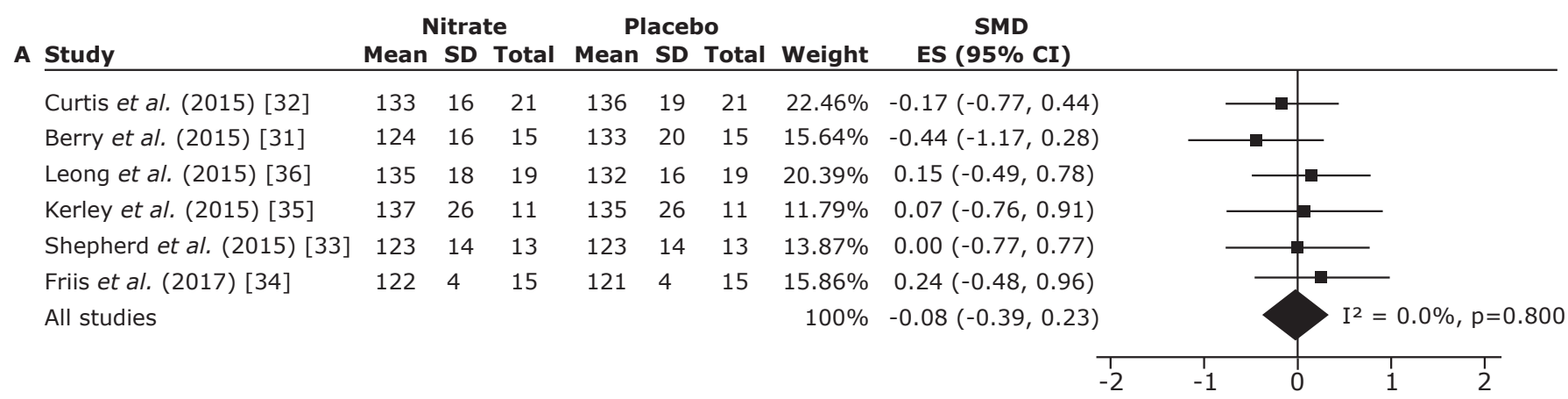

\begin{tabular}{lrrrrrrrrr} 
& \multicolumn{1}{c}{ Nitrate } & \multicolumn{1}{c}{ Placebo } & \multicolumn{1}{c}{ SMD } \\
B Study & Mean & SD & Total & Mean & SD & Total & Weight & ES (95\% CI) \\
\hline Curtis et al. (2015) [32] & 77 & 9 & 21 & 81 & 13 & 21 & $26.52 \%$ & $-0.35(-0.96,0.26)$ \\
Berry et al. (2015) [31] & 77 & 10 & 15 & 81 & 10 & 15 & $19.03 \%$ & $-0.36(-1.08,0.36)$ \\
Leong et al. (2015) [36] & 79 & 12 & 19 & 79 & 12 & 19 & $24.47 \%$ & $0.01(-0.62,0.65)$ \\
Kerley et al. (2015) [35] & 72 & 12 & 11 & 81 & 12 & 11 & $13.34 \%$ & $0.72(-1.58,0.14)$ \\
Shepherd et al. (2015) [33] & 79 & 9 & 13 & 78 & 9 & 13 & $16.64 \%$ & $0.11(-0.66,0.88)$ \\
All studies & & & & & & & $100 \%$ & $-0.24(-0.55,0.08)$
\end{tabular}

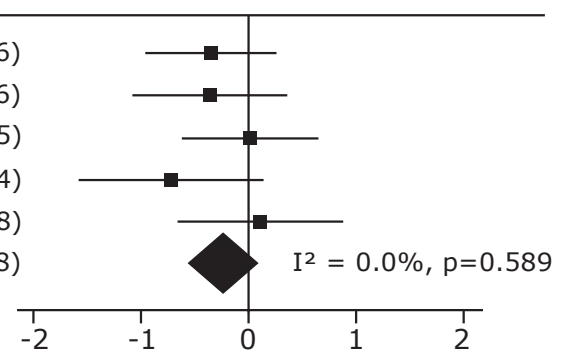

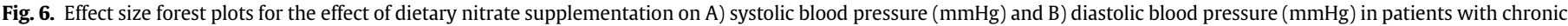
obstructive pulmonary disease.

This drop in blood pressure is the post-exercise hypotension phenomenon which has previously been reported [42-44]. During the recovery phase after exercise several mechanisms contribute to a lower blood pressure including the mediated decreases in sympathetic nerve activity, a decreased signal transduction from sympathetic nerve activation into vasoconstriction as well as local vasodilator mechanisms [45].

In the current study a dose of $\sim 8 \mathrm{mmol}$ nitrate per day was applied. This dose was based on a dose-response study that described a dose of $8.4 \mathrm{mmol} / \mathrm{L}$ was needed to significantly change oxygen parameters in recreationally active men [46]. Previous studies have used a wide range in both the amount (i.e. dose and duration) and source of nitrate supplemented and found contradictory results $[14,15,17,18]$, even in COPD $[31-36]$. In the current study we used sodium nitrate as nitrate-rich supplement, since we were interested in the effects of nitrate without needing to account for unknown interactions with other interventional compounds in the solution. Furthermore, sodium nitrate also reduced the oxygen costs of exercise and lowered the blood pressure in healthy adults [47-49]. Nevertheless, the current study shows that sodium nitrate does not affect mechanical efficiency and blood pressure in COPD. All previous studies in COPD used BRJ as a source of nitrate. Although beneficial effects on physiological responses of BRJ are ascribed to the high nitrate content [19], it is still possible that nitrate is not the active nutrient after all or that nitrate interacts with other compounds (e.g. vitamin C, potassium and polyphenols) in $B R J$, that cause the beneficial effects. Indeed recent studies showed greater blood pressure lowering and oxygen consumption lowering effects of BRJ, rocket salad beverage or spinach beverage compared to sodium nitrate in healthy adults [50,51]. However, the metaanalysis performed in the current study shows no beneficial effect of BRJ on blood pressure in patients with COPD. Therefore, the results of the current study suggest no beneficial effect of dietary nitrate on blood pressure in patients with COPD and question the efficacy on oxygen consumption and exercise performance.

In the current study the effect of sodium nitrate intake on cardiac biomarkers Hs-TNT, NT-proBNP and CK was investigated. These cardiac biomarkers are known biomarkers for the diagnosis of myocardial injury and heart failure, are associated with increased risk of cardiovascular and all-cause mortality and are known to increase following exercise $[52,53]$. In the current study we indeed show elevated levels of the cardiac markers after the cycling test, however, the changes were unaffected by sodium nitrate ingestion. Note that the observed higher Hs-TNT levels at the first blood sampling (early in the morning) can be ascribed to the diurnal rhythm of Hs-TNT [54]. This study is also one of the first studies investigating the effect of sodium nitrate on skin and core temperature. Only the effect of BRJ on oxygen cost of desert marching was previously investigated and reported an elevated rise in core temperature after nitrate ingestion without a change in skin temperature [55]. Based on the expected vasodilatory effect of NO we expected that core and skin temperatures would have been affected. However, no changes in both body temperatures were observed. More studies are needed to investigate the acute as well as the longer term effects of dietary nitrate on cardiac markers and body temperatures.

In conclusion, both acute and 7-days sodium nitrate supplementation does not increase mechanical efficiency, lower blood pressure and modulate cardiac markers of mild-to-moderate patients with COPD. Dietary nitrate does not seem to be a promising adjunct to PR to enhance the effects of exercise training.

\section{Statement of authorship}

Designed research: $C B, L V, L v L$ and AS; conducted research; RB and $\mathrm{SH}$; analyzed data: $\mathrm{RB}, \mathrm{BK}, \mathrm{SM}$ and $\mathrm{AS}$; wrote paper: RB, HG and AS; had primary responsibility for final content: $\mathrm{RB}, \mathrm{SH}, \mathrm{CB}, \mathrm{BK}, \mathrm{LV}$, LvL, SM, HG and AS.

\section{Funding sources}

This study was financially supported by a fellowship of The European Society for Clinical Nutrition and Metabolism awarded to Coby van de Bool, and by Nutricia Research and Lung Foundation Netherlands (grant: 3.4.12.023). 


\section{Conflict of interest}

The authors declare no conflicts of interst.

\section{Acknowledgements}

The authors would like to thank all the patients that participated in this study.

\section{Appendix A. Supplementary data}

Supplementary data related to this article can be found at https://doi.org/10.1016/j.clnu.2017.10.011.

\section{References}

[1] Vogelmeier CF, Criner GJ, Martinez FJ, Anzueto A, Barnes PJ, Bourbeau J, et al. Global strategy for the diagnosis, management, and prevention of chronic obstructive lung disease 2017 report. GOLD executive summary. Am J Respir Crit Care Med 2017;195:557-82.

[2] Chen W, Thomas J, Sadatsafavi M, FitzGerald JM. Risk of cardiovascular comorbidity in patients with chronic obstructive pulmonary disease: a systematic review and meta-analysis. Lancet Respir Med 2015;3:631-9.

[3] Cebron Lipovec N, Beijers RJ, van den Borst B, Doehner W, Lainscak M, Schols AM. The prevalence of metabolic syndrome in chronic obstructive pulmonary disease: a systematic review. Copd 2016;13:399-406.

[4] Beijers RJ van de Bool C, van den Borst B, Franssen FM, Wouters EF, Schols AM. Normal weight but low muscle mass and abdominally obese: implications for the cardiometabolic risk profile in chronic obstructive pulmonary disease. J Am Med Dir Assoc 2017;18:533-8.

[5] van den Borst B, Gosker HR, Schols AM. Central fat and peripheral muscle: partners in crime in chronic obstructive pulmonary disease. Am J Respir Crit Care Med 2013;187:8-13.

[6] Baarends EM, Schols AM, Akkermans MA, Wouters EF. Decreased mechanical efficiency in clinically stable patients with COPD. Thorax 1997;52:981-6.

[7] Franssen FM, Wouters EF, Baarends EM, Akkermans MA, Schols AM. Arm mechanical efficiency and arm exercise capacity are relatively preserved in chronic obstructive pulmonary disease. Med Sci Sports Exerc 2002;34: 1570-6.

[8] Richardson RS, Leek BT, Gavin TP, Haseler LJ, Mudaliar SR, Henry R, et al. Reduced mechanical efficiency in chronic obstructive pulmonary disease but normal peak VO2 with small muscle mass exercise. Am J Respir Crit Care Med 2004;169:89-96.

[9] Rabinovich RA, Vilaro J. Structural and functional changes of peripheral muscles in chronic obstructive pulmonary disease patients. Curr Opin Pulm Med 2010;16:123-33.

[10] van den Borst B, Slot IG, Hellwig VA, Vosse BA, Kelders MC, Barreiro E, et al. Loss of quadriceps muscle oxidative phenotype and decreased endurance in patients with mild-to-moderate COPD. J Appl physiology 2013;114:1319-28.

[11] Remels AH, Schrauwen P, Broekhuizen R, Willems J, Kersten S, Gosker HR, et al. Peroxisome proliferator-activated receptor expression is reduced in skeletal muscle in COPD. Eur Respir J 2007;30:245-52.

[12] Lundberg JO, Weitzberg E, Gladwin MT. The nitrate-nitrite-nitric oxide pathway in physiology and therapeutics. Nat Rev Drug Discov 2008;7:156-67.

[13] Guyton A, Hall J. Textbook of medical physiology. 11th ed. Philadelphia: Elsevier Saunders; 2016.

[14] Siervo M, Lara J, Ogbonmwan I, Mathers JC. Inorganic nitrate and beetroot juice supplementation reduces blood pressure in adults: a systematic review and meta-analysis. J Nutr 2013:143:818-26.

[15] Ashor AW, Lara J, Siervo M. Medium-term effects of dietary nitrate supplementation on systolic and diastolic blood pressure in adults: a systematic review and meta-analysis. J Hypertens 2017;35:1353-9.

[16] Jones AM. Dietary nitrate supplementation and exercise performance. Sports Med 2014;44(Suppl. 1):S35-45.

[17] McMahon NF, Leveritt MD, Pavey TG. The effect of dietary nitrate supplementation on endurance exercise performance in healthy adults: a systematic review and meta-analysis. Sports Med 2017;47:735-56.

[18] Pawlak-Chaouch M, Boissiere J, Gamelin FX, Cuvelier G, Berthoin S, Aucouturier J. Effect of dietary nitrate supplementation on metabolic rate during rest and exercise in human: a systematic review and a meta-analysis. Nitric Oxide Biol Chem 2016;53:65-76.

[19] Lansley KE, Winyard PG, Fulford J, Vanhatalo A, Bailey SJ, Blackwell JR, et al. Dietary nitrate supplementation reduces the $\mathrm{O} 2$ cost of walking and running: a placebo-controlled study. J Appl Physiology 2011;110:591-600.

[20] Hoon MW, Johnson NA, Chapman PG, Burke LM. The effect of nitrate supplementation on exercise performance in healthy individuals: a systematic review and meta-analysis. Int J Sport Nutr Exerc Metabolism 2013;23:522-32.

[21] Wasserman K, Hansen JE, Shue DY, Whipp BJ. Principles of exercise testing and interpretation. Philadelphia: Lea and Febiger; 1987.
[22] Govoni M, Jansson EA, Weitzberg E, Lundberg JO. The increase in plasma nitrite after a dietary nitrate load is markedly attenuated by an antibacterial mouthwash. Nitric Oxide Biol Chem 2008;19:333-7.

[23] Ettema G, Loras HW. Efficiency in cycling: a review. Eur J Appl Physiology 2009;106:1-14.

[24] Weir JB. New methods for calculating metabolic rate with special reference to protein metabolism. J Physiology 1949;109:1-9.

[25] Cermak NM, Hansen D, Kouw IW, van Dijk JW, Blackwell JR, Jones AM, et al. A single dose of sodium nitrate does not improve oral glucose tolerance in patients with type 2 diabetes mellitus. Nutr Res 2015;35:674-80.

[26] Hedges L. Distribution theory for Glass's estimator of effect size and related estimators. J Educ Behav Stat 1981;6:107-28.

[27] Kelly J, Fulford J, Vanhatalo A, Blackwell JR, French O, Bailey SJ, et al. Effects of short-term dietary nitrate supplementation on blood pressure, $\mathrm{O} 2$ uptake kinetics, and muscle and cognitive function in older adults. Am J Physiology Regul Integr Comp Physiology 2013;304:R73-83.

[28] Siervo M, Oggioni C, Jakovljevic DG, Trenell M, Mathers JC, Houghton D, et al. Dietary nitrate does not affect physical activity or outcomes in healthy older adults in a randomized, cross-over trial. Nutr Res 2016;36:1361-9.

[29] Hirai DM, Zelt JT, Jones JH, Castanhas LG, Bentley RF, Earle W, et al. Dietary nitrate supplementation and exercise tolerance in patients with heart failure with reduced ejection fraction. Am J physiology Regul Integr Comp Physiology 2017;312:R13-22.

[30] Shepherd AI, Gilchrist M, Winyard PG, Jones AM, Hallmann E, Kazimierczak R, et al. Effects of dietary nitrate supplementation on the oxygen cost of exercise and walking performance in individuals with type 2 diabetes: a randomized, double-blind, placebo-controlled crossover trial. Free Radic Biol Med 2015;86: 200-8.

[31] Berry MJ, Justus NW, Hauser JI, Case AH, Helms CC, Basu S, et al. Dietary nitrate supplementation improves exercise performance and decreases blood pressure in COPD patients. Nitric Oxide Biol Chem 2015:48:22-30.

[32] Curtis KJ, O'Brien KA, Tanner RJ, Polkey JI, Minnion M, Feelisch M, et al. Acute dietary nitrate supplementation and exercise performance in COPD: a doubleblind, placebo-controlled, randomised controlled pilot study. PloS One 2015;10, e0144504.

[33] Shepherd AI, Wilkerson DP, Dobson L, Kelly J, Winyard PG, Jones AM, et al. The effect of dietary nitrate supplementation on the oxygen cost of cycling, walking performance and resting blood pressure in individuals with chronic obstructive pulmonary disease: a double blind placebo controlled, randomised control trial. Nitric Oxide Biol Chem 2015;48:31-7.

[34] Friis AL, Steenholt CB, Lokke A, Hansen M. Dietary beetroot juice - effects on physical performance in COPD patients: a randomized controlled crossover trial. Int J Chronic Obstr Pulm Dis 2017;12:1765-73.

35] Kerley CP, Cahill K, Bolger K, McGowan A, Burke C, Faul J, et al. Dietary nitrate supplementation in COPD: an acute, double-blind, randomized, placebocontrolled, crossover trial. Nitric Oxide Biol Chem 2015;44:105-11.

[36] Leong P, Basham JE, Yong T, Chazan A, Finlay P, Barnes S, et al. A double blind randomized placebo control crossover trial on the effect of dietary nitrate supplementation on exercise tolerance in stable moderate chronic obstructive pulmonary disease. BMC Pulm Med 2015;15:52.

37] Muggeridge DJ, Sculthorpe N, Grace FM, Willis G, Thornhill L, Weller RB, et al. Acute whole body UVA irradiation combined with nitrate ingestion enhances time trial performance in trained cyclists. Nitric Oxide Biol Chem 2015;48: $3-9$.

[38] Gosens W, Van't Hul AJ, Oomen JM, Hesselink MK, Borghouts LB. Mechanica efficiency in chronic obstructive pulmonary disease. J Cardiopulm Rehabilitation Prev 2017;37:146-53.

39] Bondonno CP, Liu AH, Croft KD, Ward NC, Shinde S, Moodley Y, et al. Absence of an effect of high nitrate intake from beetroot juice on blood pressure in treated hypertensive individuals: a randomized controlled trial. Am J Clin Nutr 2015;102:368-75.

[40] Kerley CP, Dolan E, Cormican L. Nitrate-rich beetroot juice selectively lowers ambulatory pressures and LDL cholesterol in uncontrolled but not controlled hypertension: a pilot study. Ir J Med Sci 2017;186:895-902.

[41] Siervo M, Lara J, Jajja A, Sutyarjoko A, Ashor AW, Brandt K, et al. Ageing modifies the effects of beetroot juice supplementation on 24-hour blood pressure variability: an individual participant meta-analysis. Nitric Oxide Bio Chem 2015;47:97-105.

[42] Halliwill JR. Mechanisms and clinical implications of post-exercise hypotension in humans. Exerc Sport Sci Rev 2001;29:65-70.

[43] Hamer M. The anti-hypertensive effects of exercise: integrating acute and chronic mechanisms. Sports Med 2006:36:109-16.

[44] MacDonald JR. Potential causes, mechanisms, and implications of post exercise hypotension. J Hum Hypertens 2002;16:225-36.

[45] Halliwill JR, Buck TM, Lacewell AN, Romero SA. Postexercise hypotension and sustained postexercise vasodilatation: what happens after we exercise? Exp Physiol 2013;98:7-18.

[46] Wylie LJ, Kelly J, Bailey SJ, Blackwell JR, Skiba PF, Winyard PG, et al. Beetroo juice and exercise: pharmacodynamic and dose-response relationships. J Appl Physiology 2013;115:325-36.

[47] Larsen FJ, Schiffer TA, Borniquel S, Sahlin K, Ekblom B, Lundberg JO, et al Dietary inorganic nitrate improves mitochondrial efficiency in humans. Cell Metab 2011:13:149-59.

[48] Larsen FJ, Weitzberg E, Lundberg JO, Ekblom B. Effects of dietary nitrate on oxygen cost during exercise. Acta Physiol 2007;191:59-66. 
[49] Rammos C, Hendgen-Cotta UB, Sobierajski J, Bernard A, Kelm M, Rassaf T Dietary nitrate reverses vascular dysfunction in older adults with moderately increased cardiovascular risk. J Am Coll Cardiol 2014;63:1584-5.

[50] Jonvik KL, Nyakayiru J, Pinckaers PJ, Senden JM, van Loon LJ, Verdijk LB. Nitrate-rich vegetables increase plasma nitrate and nitrite concentrations and lower blood pressure in healthy adults. J Nutr 2016:146:986-93.

[51] Flueck JL, Bogdanova A, Mettler S, Perret C. Is beetroot juice more effective than sodium nitrate? The effects of equimolar nitrate dosages of nitrate-rich beetroot juice and sodium nitrate on oxygen consumption during exercise. Appl Physiol, Nutr Metab = Physiol Appliquee, Nutr Metabol 2016;41:421-9.

[52] Klinkenberg LJ, Luyten P, van der Linden N, Urgel K, Snijders DP, Knackstedt C et al. Cardiac troponin T and I Release after a 30-km run. Am J Cardio 2016;118:281-7.
[53] van der Linden N, Klinkenberg LJ, Bekers O, Loon LJ, Dieijen-Visser MP, Zeegers MP, et al. Prognostic value of basal high-sensitive cardiac troponin levels on mortality in the general population: a meta-analysis. Medicine 2016;95:e5703.

[54] Klinkenberg LJ, van Dijk JW, Tan FE, van Loon LJ, van Dieijen-Visser MP, Meex SJ. Circulating cardiac troponin T exhibits a diurnal rhythm. J Am Coll Cardiol 2014;63:1788-95

[55] Kuennen M, Jansen L, Gillum T, Granados J, Castillo W, Nabiyar A, et al. Dietary nitrate reduces the $\mathrm{O} 2$ cost of desert marching but elevates the rise in core temperature. Eur J Appl Physiology 2015;115:2557-69. 